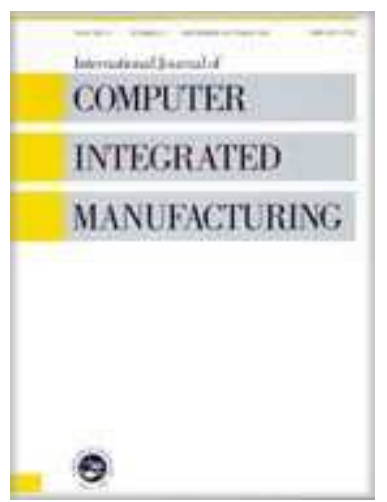

\title{
Determining numbers of workstations and operators for a linear walking-worker assembly line
}

\begin{tabular}{|r|l|}
\hline Journal: & International Journal of Computer Integrated Manufacturing \\
\hline Manuscript ID: & TCIM-2005-IJCIM-0045.R1 \\
\hline Manuscript Type: & Original Manuscript \\
\hline Author: & 22-Feb-2006 \\
\hline & $\begin{array}{l}\text { Complete List of Authors: } \\
\text { Enging, Qian; University of Bath, Department of Mechanical } \\
\text { Onginering } \\
\text { Mileham, Antony; University of Bath, Department of Mechanical } \\
\text { Engineering }\end{array}$ \\
\hline Keywords: & $\begin{array}{l}\text { MODELLING, PERFORMANCE ANALYSIS, ASSEMBLY LINES, } \\
\text { SIMULATION APPLICATIONS, MANUFACTURING SYSTEMS, } \\
\text { FLEXIBLE ASSEMBLY }\end{array}$ \\
\hline Keywords (user): & WALKING WORKERS \\
\hline &
\end{tabular}

\section{S ScholaroNE" \\ Manuscript Central}




\title{
Determining numbers of workstations and operators for a linear walking-worker assembly line
}

\author{
QIAN WANG*, GERAINT WYN OWEN and ANTONY ROY MILEHAM \\ Department of Mechanical Engineering, University of Bath, Bath, BA2 7AY, UK \\ E-mail: A.R.Mileham@bath.ac.uk, G.W.Owen@bath.ac.uk, Q.Wang@bath.ac.uk \\ *Corresponding author. Email: Q.Wang@,bath.ac.uk
}

\begin{abstract}
This paper presents an investigation into a system of linear walking-worker (WW) assembly lines that have been implemented in a local SME (small and medium enterprise). The work aims to observe the relevant impact on logical interactions and interrelationships between the number of workstations and the number of walking workers against the system performance and to minimise these numbers providing a quick response in re-configuration of the system to meet a given output and cycle time. This has been achieved by developing a simulation model for a theoretical study into the linear WW system using a manufacturing-focused simulation tool. By examination of the results of a series of simulation experiments, a minimisation of the numbers of workstations and walking workers for a linear WW line configuration can be quantified. The research concluded that this multi-skilled linear WW assembly line is able to sustain a higher flexibility and efficiency as compared to a conventional linear fixedworker (FW) assembly line under similar conditions.
\end{abstract}

\section{Keywords}


Walking workers, assembly lines, simulation, flexible assembly, modelling

\section{Introduction}

A typical manual assembly line has separate workers performing assembly tasks at each workstation whilst assembled items are moved on a conveyor system from workstation to workstation. The operating times at each workstation are expected to be almost equal in order to achieve a balanced assembly system. These times are highly contingent on the variation of speed or skill of the workers and the processes used at each workstation. The slowest worker often dictates the line output and the production rate, which are also affected and controlled by the workstation with the longest processing time; nevertheless, this longest processing time also determines the level of line balance. In most conventional manual assembly lines, there is one (or more) seated or standing assembly worker who repeatedly performs a single assembly task at each workstation (or machine) and when this task has been accomplished, the assembled part will be passed on to the next assembly worker at a downstream workstation for the next assembly task to be done. This procedure will carry on until all the assembly work has been completed. In this article, we name this type of serial production line with one stationary worker per workstation as a linear fixed-worker (FW) assembly line, i.e., an assembly worker always stays at a fixed-position workstation to do a single and often repetitive assembly work along a sequential production line. One of the main drawbacks for this type of production line is the difficulty of 'in-process' line balancing. Any variance at any link in a theoretically balanced production chain will result in disruptions in production and could even stop the entire production line. Because each 
workstation needs an assembly worker, the number of operators is normally equal to (or greater than to cover breaks etc.,) the number of workstations regardless of the volume of production. This is wasteful when the production volume is relatively low as the production line still has to be fully staffed at each workstation leading to a poor efficiency, responsiveness and the system's re-configuration flexibility to a varying demand of outputs. Moreover, the variation in individual working speed for each worker increases the complexity of balancing the workload of individuals in terms of percentage utilisation. To deal with this line-balancing problem, the most commonly used approach is to add appropriate buffers into the production line. This simply increases unnecessary costs and work-in-process (WIP). Previous work (Mileham et al. 2000) outlines that fixed-fitter lines tend to have low flexibility (in terms of fitters and products), need constant demand and can be difficult to balance.

The fundamental distinction for the definition of linear WW assembly lines compared to the definition of conventional linear FW assembly lines is that each worker is crosstrained so that they are capable of assembling each product completely from beginning to end and do this by walking from workstation to workstation along the production line. Unlike a linear FW line where each worker is permanently engaged at one workstation, in a linear WW line, each worker travels with his / her own assembled item downstream and stops at each workstation to carry out the essential assembly work as scheduled. When a walking worker finishes the assembly of a product, the worker walks back to the front end of the production line to start the assembly of another and the above-described procedure is repeated. This method attempts to combine some advantages that originate from a workbench system or a cellular system with its high 
flexibility in terms of product variability and from a linear FW assembly line with its high efficiency in terms of high output. Some advantages of using the linear WW line by application in a local company include: more easy line balancing and a reduced buffer requirement, a greater tolerance of work time variations, adjustability of the number of operators in response to the output requirement, thereby minimising labour costs, cultural changes and an improvement of human factors such as motivation, accountability and responsibility. The main drawbacks are that each assembly worker must be fully trained to complete the assembly of a whole product. It may be expensive or difficult to train every worker for a large number of operations, as only relatively low-skilled workers are available (Bischak 1996 and Zavadlav et al. 1996). Hence, the suitability of implementing the WW system largely depends on the nature of assembled products as well as the level of cross-training. Clearly, the human factors (such as uneven skills of workers) rather than machines may limit the rate of output. However, despite having many advantages, the linear WW assembly line is not widely implemented in industry. It is thought that this is due to a lack of supportive theories and methodology to underpin real manufacturing applications.

\section{Literature studies}

There are a small number of publications in the literature relating to WW assembly systems. A report (Anon 1985) introduced that several Japanese companies, for instance, Toyota Motor Co., trained each worker to perform all the jobs in a workshop. These so-called multi-functional workers were capable of rotating from job to job at every process within the workshop. In some cases, workers were rotated among the 
different processes of an assembly line and thus contributed to greater system efficiency and workforce flexibility. It was reported by observation that multi-functional workers were better able to respond to alterations in cycle times, operation routines and contents of individual jobs as the demand changed. It was also observed that they were more attentive and were better able to spot problems, thereby increasing improvement possibilities and decreasing the frequency of workplace accidents. However, this onepage paper does not cover any more details regarding to how those multi-functional workers were organised to rotate among machines in the workshop and how they performed their jobs. Based on the premise that those companies just trained their workers to be able to operate more than one machine; this is not the same as a linear WW assembly line as defined in this paper.

Line balancing is a major problem for conventional assembly lines. Black and Schroer (1997) reported that having moving multi-functional workers in a U-shaped line permitted rapid re-balancing in production. They suggested that for such a line the processing or operation time at each workstation would not have to be precisely balanced, compared to a conventional FW line. They argued that this was because the balance could be achieved by having the workers who walk from workstation to workstation as long as the sum of the operation times for each worker was approximately equal. This result was based on a case study in the apparel industry that employed multi-functional workers who moved between sewing machines in a cell with great variations in processing time. In this cell, each worker covered at least two or more machines, and they followed each other around the cell in a so-called rabbit chase. All sewing machines in the cell were laid out in a U-shaped line to minimise walking 
distances between machines. Due to the physical restriction of this U-shaped design in which only a limited number of workers (between 3 and 5) were possible to work amongst 13 stations, both operator and workstation utilisations presented in his paper were therefore low. The results of their research did not show a higher performance by using moving workers in a U-shaped line over a conventional assembly line. Although the authors claimed that by having a worker travelling among sewing machines appears to accommodate the extremely unbalanced system more easily, there was no further theoretical analysis or simulation result provided to support this claim.

Nakade and Ohno $(1997,1999,2003)$ developed a rather simplified mathematical model of a U-shaped production line with single or multiple, multi-functional workers to determine the number of workers required for a given output while minimising the overall cycle time. The development of this model had to be restrained by defining the upper and lower bounds for an expected cycle time. These bounds were used to help determine the approximate values for the cycle time. Furthermore, it is generally accepted that mathematically modelling techniques often encounter difficulties when modelling a detailed manufacturing system (Wang and Chatwin 2003).

Bartholdi et al (1999) gives a rather interesting name of organising walking workers in a way, which is called 'bucket brigade'. Within a 'bucket brigade' system, walking workers follow a simple rule: each worker carries work forward from station to station until someone takes over your work; then this worker goes back for taking the work from his/her predecessor. This can be explained further that when the last worker completes a product, he/she walks back upstream and takes over the work of his/her 
predecessor, who then walks back and takes over the work of his/her predecessor, and so on until the first worker begins a new product at the start of the line. The main benefit reported by Bartholdi et al (2001) is that this simple functioning protocol yielded a spontaneous line balancing, i.e., a bucket brigade line has self-balancing capability as it absorbs variance in the work by moving the workers where the work is. However, this claim has led to a debate by a few researchers with completely opposite views on the possibility of this distinctive and valuable feature that could be achievable. For instance, Bratcu and Dolgu (2005)'s paper is mainly based on a literature study of the research work made by Bartholdi. It concludes that under certain conditions, a bucket brigade system can reach a steady state (constant cycle time) with a maximal production rate because all the workers are fully occupied in this configuration therefore no blocking, no waiting and no idle times. However, this statement contradicts to one of Bartholdi et al (2001)'s own papers that a worker can be blocked in a bucket brigade system. By contrast, Zavadlav et al (2001) raised a number of queries regarding to the studies by Bartholdi et al. He argued that the Bartholdi's research outcome was based on simplified mathematical models that did not consider randomness in any form including no variability in processing times. Even Bartholdi et al (2001) admitted in their paper that the main weakness of their models is that it treats workers simplistically in describing each as merely a velocity function. Another major query was that how this bucket brigade system can be operated by simply organising walking workers through a single management rule; and whether workers would resist this rather stressful relaybaton-like production manner in practice. Zavadlav et al (2001) suggested that this system can exhibit chaotic behaviour and the positions of the workers may change in an unpredictable and unstable way. 
Ghinato and Fujii et al (1998) developed a so-called spacefilling curve-based algorithm to minimise the number of multi-functional workers within a given cycle time. The basic idea for this approach is to generate a curve that visits all machines along a Ushaped layout starting from entrance or exit of the production line. The curve visits the machines moving forward from one machine to one of its adjacent machines in a given routing pattern. To find the optimum number of assigned workers, starting from the assignment of the first worker, it computes whether the operating time of machine 1 is less than the specified cycle time, i.e., is the routine time of worker A less than the cycle time? If so, then the procedure moves to the next machine along the curve and tests again whether the new routing time is still less than the cycle time and so on until no machine can be added to the worker's routine. Then, the assignment of worker B begins. This procedure goes on until the last machine is reached and assigned to a worker. However, this is a rather simplified algorithm; the model used is assumed to be deterministic only with all parameters known. Nevertheless, the optimisation of the number of walking workers using the above method simply ignores other factors such as how this assignment affects the overall system performance in terms of for example measures of output and labour utilisation.

In other aspects, Celano and Antonio et al's (2003) research was focused on the 'human factors' issues. They proposed a simple 'help policy' algorithm (or rule) for a manual mixed model (product) U-shaped assembly line with partial walking workers. They defined a worker as a 'critical worker' when this worker was unable to cope with current tasks at a workstation during the production cycle time. If this is the case, a rule 
called the 'Downstream Worker Help Policy and Frontal Worker Help Policy' must be strictly followed by running to help this person if available, thereby, minimising the total conveyor stoppage time.

Mileham and Owen et al (2000) empirically implemented a rather large-scale system of linear WW lines into a local medium-sized manufacturing company where a conventional workbench assembly system had been dominant and used for many years. The company produces 8 major models in similarity with a high level of customisation. Because the capacity of the original system (the workbench system) was no longer sufficient to deal with either the current or future demand; the solution was to re-design the old system to be 3 parallel linear WW assembly lines. To implement this system, a new hybrid production control system that combined Kanban, Reorder Point Control (RPC) and MRP had also been introduced and made operational following a culture change through teamwork and multiple skill training. This combined strategy was reported to have achieved good results by significantly reducing costs, improving quality of products and increasing productivity.

To enable a much better understanding of the factors that affect linear WW assembly lines, research on the implemented system described above has continued by analysing existing data, developing a simulation model of existing linear WW lines and then conducting experiments under varying configurations using Witness ${ }^{\circledR}$ to observe the system behaviour. This paper summarises the work that involves characterising and quantifying the effect of the factors (i.e., numbers of workstations and operators) that influence the system performance of the linear WW line and understanding the critical 
relationships between these factors. This has been achieved based on the simulation results that were obtained from the established WW models for the system of 3 linear WW assembly lines, which are detailed below.

\section{The system of linear WW assembly lines}

Figure 1 shows a snapshot of the simulation model of the linear WW assembly system for a local medium-sized manufacturing company. It consists of 3 parallel assembly lines each producing one of 3 families of products (named as A, B, C series), and one parallel 'prepare-zone' line, starting from the sign of 'Wait Here' (indicated by an arrow in the middle). In the 'prepare zone' line, job loaders prepare and categorise assembly instruction paperwork (job sheets) received from the shop floor controller (near the sign of 'Wait Here') who downloads them from a Central Production Control System (CPCS) on a daily basis (all job sheet barcodes have previously been scanned by the CPCS for component availability). The job loader places all the pre-assembled components along with all associated job sheets and operational guidelines onto a pallet. These components on pallets then queue on the 'prepare-zone' conveyor waiting to be picked and assembled by the next free walking worker. Each loaded pallet is labelled by a different colour card, which helps walking workers distinguish different catalogues of products to be assembled on their own production lines. The entire conveyor system is a non-powered roller conveyor therefore assembly workers need to move the loaded pallets stopping by each workstation by pushing them on the conveyor. This design gives flexibility to individual workers to move the queuing pallets whenever they need. 
Each linear WW assembly line (named as A, B and C) has a flexible number of at least 4 walking workers and operates under similar operating conditions. Each line is balanced depending on the products being assembled. Table 1 shows the system parameters used for the system model. Since the nature of operation procedures for the three parallel lines is similar, the research work was based on a case study from Line A and expended experimental models under various scenarios were also based on Line A. A brief operation procedure at workstations is described below:

[Insert figure 1 about here]

Each workstation is stocked or equipped with the required components, tooling and procedures from line-side shelves. A walking worker walks to the front end of the 'prepare zone' (conveyor) and selects the next working pallet using the gravity roller track to move the pallet into the first assembly workstation. The specific activities of each workstation are described in Process Instruction Sheets on each pallet. The walking worker moves with the pallet stopping at each workstation and assembling the product in accordance with the appropriate Process Instruction Sheet. On completion at the last workstation, the walking worker pushes the finished product onto the test rig section to await testing. The walking worker then returns to the front end of the 'prepare zone' to pick the next product to be assembled.

[Insert table 1 about here] 


\subsection{Simulation models}

Experimental simulation models for various configurations in terms of numbers of workstations and workers for Line A have been created, verified and validated. These models also include a baseline simulation model for a comparable linear FW assembly line that has been created and used to compare the system performance with that of the linear WW line. The animation facilities within the Witness ${ }^{\circledR}$ simulation system were used to provide visual insight about material flows, WIP levels, bottlenecks and observing WW movement.

Some assumptions, systems' properties and key points for the developed models are summarised below:

1. Production operates without machine failures or similar malfunctions on the assembly line during the certain period of production ( 7 hours), i.e., the workers, rather than machines, are the bottleneck;

2. All processing time distributions (i.e., work times) with various standard deviations are identified; thereby the overall cycle time of the assembly line can be determined. The value of the mean processing time at each workstation is different as a complete balance was not possible but they fall within a range of 276 seconds to 324 seconds. Despite the fact that each walking worker was crossed-trained to complete a single operation at each workstation within a certain period of time, the working speed of individuals may slightly vary from time to time due to fatigues or uneven skilled levels. As a consequence, it was determined that the processing times follow the 
normal distribution with a range of standard deviations (SD) from $\pm 2.0 \%$ to \pm 11.5 $\%$ of the mean times as input for the simulation models;

3. The walking distance in terms of time is evenly distributed between two adjacent workstations for all walking workers. Although the time spent on walking compared to the time spent on processing at each workstation is relatively small for the current system, the walking time has been modelled into the WW simulation models;

4. There is an infinite supply of sub-assembled components on the line-side shelves along the production line from the first workstation to the last workstation, implying that workstations never starve because of component shortages; all finished products are removed from the line immediately; therefore, they will not block the end of the line due to factors such as insufficient storage;

5. To avoid collision, two walking workers should not occupy the same workstation (i.e., a faster walking worker will not overtake a slower walking worker). If a previous worker has not completed the assembly work at a workstation, the worker behind must wait before entering this workstation until the job has been completed and the workstation is empty;

6. There are limited buffer spaces (i.e., the conveyor) between workstations to enable a worker and product to stop on reaching a full workstation. When the waiting worker becomes unblocked they continue and the product is assembled on reaching the clear workstation as scheduled;

\section{Simulation result and analysis}


Extensive simulation results have been produced from the experimental models and these have been analysed to measure the system performance.

4.1. Effect of the number of walking workers and workstations on the line output

The daily output of this implemented linear WW line is a key measure of system performance. Figure 2 indicates that the line output as a function of the number of walking workers $\mathrm{k}$ on an $\mathrm{n}$-workstation (indicated as $\mathrm{n}$ WKs, where $\mathrm{n}$ varies from 1 to 10) line. Obviously, by increasing the number of walking workers $k$ on an n-workstation line the overall line output will increase rapidly until this output reaches the maximum value, where $\mathrm{k}=\mathrm{n}$. After this, adding additional walking workers to a linear WW line will not increase the line output unless additional workstations are added to the line. This can be simply explained by the fact that any additional workers cannot be utilised at the same time if there is no spare workstation for them to work as all workstation are fully engaged. This is the same situation as a linear FW line where the line is fully manned. On the other hand, it can be seen that the linear WW line has ability (as well as flexibility) to easily adjust the output rate by simply adding or removing walking workers from the line to reflect the daily demand. By contrast, a linear FW line does not have this characteristic as all workstations must be fully staffed and the line has to run at full capacity even for a low volume.

Figure 2 also shows a bunch of linear overlapping curves which represent the overall output as a function of the number of walking workers $\mathrm{k}$ for an $\mathrm{n}$-workstation line where $\mathrm{k} \leq \mathrm{n}$. This can be clearly seen in figure 3 . 
[Insert figure 2 about here]

Figure 3 shows a fluctuation of line outputs versus the number of workstations $n$ for varying numbers of walking workers $\mathrm{k}$ (indicated as $\mathrm{k}$ WWs, where $\mathrm{k}$ varies from 1 to 10). These fluctuations actually reflect the overlapping curves shown in figure 2 . It is interesting to observe that when $\mathrm{k}=\mathrm{n}$ where the line output reaches the maximum value, adding more workstations into the line without increasing the number of walking workers $\mathrm{k}$ will not significantly change the value of the maximum output but it will result in a slight fluctuation around this value. This fluctuation is caused by the fact that adding additional workstations to the line will actually add additional work time variations to the line. This is due to the fact that the stretched assembly process on the extended line leads to a fluctuating variance of work time variations at each workstation (note: adding more workstations to the line will not increase the overall cycle time used for a product assembly as the remaining workers will simply decrease their proportion of the total processing time at each workstation). However, this fluctuation will not make a significant impact on the value of the maximum output in this case study due to the fact that the work times at each workstation have been relatively well balanced and the variations (in terms of standard deviations $\pm \sigma$ ) for work times at each workstation is set to be relatively small. A case study for both balanced and unbalanced linear WW lines with high work time variations is reported by the authors (Wang et al 2005) in another paper.

[Insert figure 3 about here] 
Another effect, which may possibly cause this fluctuation and may also increase the overall cycle time as well as WIP, is an increasing amount of walking time. Because adding more workstations will actually increase the length of the production line thereby increasing the total amount of walking time, this may affect the maximum output as each walking worker spends too much time on walking rather working along the line. Predictably, the value of the maximum output may gradually decline if the total walking distance is designed to be too long. Since the total amount of walking time in this case study is very small compared to the overall cycle time of the production line, this variation caused by walking time appears not to be a major factor causing this fluctuation. In brief, it is believed that the degree of fluctuation is dependent on factors including the degree of work time variation and the proportion of time spent on walking.

It is also interesting to observe from figure 3 that when a linear WW line has the same number of walking workers and workstations, i.e., $\mathrm{k}=\mathrm{n}$ where the line produces a maximum output, adding one more workstation (i.e., $\mathrm{n}=\mathrm{k}+1$ ) to the line will increase the value of this maximum output and then this value becomes stable with a fluctuation as stated above. It appears to be a trend that this little gain of the increased maximum output becomes significant for a large-scale linear WW line; this can be clearly seen in table 2. In other words, this result can also be interpreted as to achieve a maximum line output; the total number of walking workers required $\mathrm{k}$ can be less than the total number of workstations $\mathrm{n}$, i.e., $\mathrm{k}<\mathrm{n}$, compared to linear FW lines, the maximum output can only be achieved by the fully manned line, where, $k=n$. 
4.2. Effect of numbers of walking workers and workstations against utilisations

The utilisation of a walking worker during production was also one key measure that reflects the system performance. For this case study, the utilisation of a walking worker can be defined as the percentage of time that this worker has been used for a product assembly.

Figures 4 and 5 illustrate a situation in which the number of walking workers $\mathrm{k}$ and the number of workstations $\mathrm{n}$ together affect the utilisation of each walking worker. It can generally be seen in figures 4 and 5 that increasing the number of walking workers $\mathrm{k}$ on an n-workstation (i.e., indicated as $n$ WKs, where $n$ varies from 1 to 10) line will decrease the percentage utilisation for each walking worker. By contrast, increasing the number of workstations $\mathrm{n}$ to a $\mathrm{k}$ WW (i.e., indicated as $\mathrm{k}$ WWs, where $\mathrm{k}$ varies from 1 to 9) line will significantly increase the utilisation of each walking worker.

Further examination of the labour utilisation as illustrated in figure 4 shows that by adjusting the number of walking workers from $\mathrm{k}$ to 1 , where $\mathrm{k} \leq \mathrm{n}$, there will be a small change in labour utilisations, which are in excess of $91 \%$ in all cases. The percentage of labour utilisation only starts to decline sharply when superfluous walking workers are added to the line, i.e., $\mathrm{k}>\mathrm{n}$, where the line output reaches the maximum value and will remain unchanged as discussed in section 4.1. For instance, the utilisation of every walking worker is from $91.03 \%$ to $100 \%$ for a 10 -workstation line if $\mathrm{k} \leq 10$ and from $96.76 \%$ to $100 \%$ for a 2 -workstation line if $\mathrm{k} \leq 2$; adjusting the number of walking 
workers $\mathrm{k}$ in the line from $\mathrm{n}$ to 1 will not significantly affect this high percentage for each labour utilisation as the overall line output will decreases as discussed in section 4 . This suggests that the linear WW line can achieve a high labour utilisation even if the overall line output decreases. Apparently, this cannot be done from a linear FW line where the adjustability of staffing levels is very limited.

[Insert figure 4 and 5 about here]

By examining the simulation results shown from figure 2 to 5 , the system designer can determine the numbers of walking workers and workstations for different configurations and scales of linear WW lines. If we take for example a requirement for an output of 50 units / day, it can be determined from figure 2 (or 3) that this output can be achieved by a minimum of 7 walking workers on a 7, 8, 9, or 10-workstation line. Examination of figure 4 , however, reveals that the utilisation of workers is higher with 8 workstations than with 7 and there is no significant gain in utilisation by going to 9 or 10 workstations. Further, as discussed as section 4.1, it can be seen in figure 3 that there is a small gain in output which can be raised to 52 units / day by going to 8 workstations and then the value of this maximum output will remain stable with a fluctuation of between 50 and 52 units / day. As a result, it can be recommended that the proposed linear WW line can be configured as a minimum number of 7 walking workers in an 8workstation line. For another example, assume that if each walking worker is required to be $80 \%$ busy for a linear WW line and the line's output is expected to be 50 units per day. From figure 3 it can be seen that this output can be approximately achieved by having 7, 8, 9 or 10 walking workers in a 7-workstation line. By examination of figure 5 
it indicates a minimum number of walking workers for achieving an utilisation of $80 \%$ is 8 , hence, a linear WW line configuration with 8 walking workers in a 7 -workstation line can be drawn up.

4.3. Comparison between fixed- and walking-worker lines

Figures 2 and 3 also provide a comparison in variation of daily outputs against the numbers of workers $\mathrm{k}$ and workstations $\mathrm{n}$ between the linear WW line and the linear FW line. The dotted lines represent the simulation results for $\mathrm{k}$ fixed workers in an nworkstation line (where, $\mathrm{k}=\mathrm{n})$.

It can be generally seen from figures 2 and 3 that the linear WW line can provide better performance in terms of line outputs than the linear FW line, where $k=n$ and $n>3$. This superiority looks more significant if the scale of both assembly lines is large. This can be further seen in table 3. It suggests that the advantage of using walking workers, compared to using fixed workers under the similar conditions in this case study, the overall output increases from $3.6 \%$ for a 4 -workstation line to $11.4 \%$ for a 12 workstation line. It appears that this increase becomes significant if the line is large. However, as discussed in section 4, large lines will also increase the overall amount of walking times as well as the overall variation of work times in the production line. Because the total amount of walking time for a 12 -workstation line is still very small compared to the overall cycle time of the production line. Therefore, these factors did not significantly affect the better performance from the linear WW line than the linear FW line shown in table 3. 
From table 3 it can also be seen that the line output can be achieved by a linear WW line with few workers and workstations than a linear FW line in similar circumstances. For example, table 3 shows that if a linear WW line consists of $\mathrm{k}$ workers and $\mathrm{n}$ workstations, where, $\mathrm{k}=\mathrm{n}$, and $\mathrm{k}>6$, this linear WW line can achieve approximately the same output using a linear FW line that needs $\mathrm{k}+1$ workers and $\mathrm{n}+1$ workstations. It is clear that the linear WW line has superior performance over the linear FW line under similar conditions.

[Insert table 2 about here]

The research outcome also indicates that the linear FW line has a higher blocking rate than that of the linear WW line under similar circumstances. This can explain why a linear WW line outperforms a linear FW line.

In general terms, significant blocking or starving will reduce the ideal output of a production line. Within an unbuffered linear FW line, blocking and starving often takes place during production because of variation in work times at each workstation. Within an unbuffered linear WW line, starving is impossible because walking workers are never starved of work at a workstation. However, workers can be blocked behind a slow worker. Table 4 shows a simulation result of highest blocking rates for two different types of lines under similar conditions, where both lines are equipped with $\mathrm{k}$ workers in an n-workstation unbuffered system. It clearly shows that in all cases a linear FW line has a higher blocking rate than a linear WW line. It indicates that the linear WW line 
has a better absorption capacity for much of the processing (work) time variations, thereby providing superior performance to the linear FW line. This can be explained by the fact that having workers moving with assembled parts between two adjacent workstations will actually act as a buffer that unblocks the workstation for the previous worker while waiting for the upstream workstation to be empty. By contrast, in a linear FW line, if a workstation ahead is blocked, then all assembly work behind may also have to stop.

[Insert table 4 about here]

Other human factors may also be present that will reduce the effect of work time variations in linear WW lines. For example, if a slow worker is causing other assembly workers behind that worker to be slowed down, there will be a certain pressure on that individual walking worker to work faster. After an assembly worker has completed a task at a workstation, and if the next downstream workstation is still occupied while the upstream assembly worker has also completed the assembly work, this worker can walk away from the workstation in order to leave of free for the upstream worker to carry out the next assembly operation. If this occurs it will also significantly decreases the blocking rates and actually improve the real line balancing. This phenomenon can be achieved by following a rule developed by the production manager.

\section{Conclusions and discussions}


This paper reports an investigation into how the system performance is influenced by varying the numbers of workers and the numbers of workstations in a system of socalled linear WW assembly lines. Through the use of simulation, the number of walking workers and workstations for a given output can be determined. The study also compared the system performance between the linear WW line and the linear FW line. The research concludes that for a known overall cycle time in a linear WW line, increasing the numbers of walking workers and workstations together will significantly increase the line output; increasing the number of walking workers without increasing the number of workstations will not increase the line output but will decrease the utilisation of each worker. The line reaches a maximum output when the number of workers is equal to the number of workstations. Adding additional workers will not increase this maximum output unless adding additional workstations to the line; by contrast, adding one or more workstations to the line may gain a further increase in the value of this maximum output. The research outcome indicates that the linear WW line has higher flexibility and efficiency in terms of output and labour utilisation over the linear FW line under similar conditions. This superior performance includes the benefit that the linear WW line demands fewer resources (labour and facilities) than the conventional linear FW line to achieve a similar output. However, the linear WW line may lose its superiority over the linear FW line if the length of the lines becomes too long so that the increasing amount of walking time becomes so significant to affect the system performance.

A design with a minimum number of workers and workstations for a linear FW line should be interpreted as a 'ideal' value, as it is highly unlikely in practice that the ideal 
output will be achieved. This is because balancing is very difficult to achieve due to the inherent and unavoidable variability of the time required by each worker to perform a given assembly task at each workstation (Groover 2002). The research work presented in this paper has shown that the implementation of a linear WW system can be an effective approach to cope with this problem of variability, which often deoptimises linear FW lines. The research indicates that the linear WW line has a better tolerance of work time variations than the linear FW line providing alternative approach to some manufacturing companies to improve the system performance and flexibility by systematically reducing the system variations using the linear WW line.

However, an implementation of linear WW lines may largely depend on the degree of complexity of a product to be assembled, as each worker has to complete a product in its entirety along the line. The cross-training is essential if this type of line is applied. A cross-trained worker can improve the system efficiency in the form of higher output, $\underline{\text { lower inventory, shorter cycle times without significant additional investment (Hop et al }}$ 2004). However, it may experience difficulties to train every worker for a large number of manufacturing operations. To obtain relatively even skilled walking workers through training is crucial to operate the linear WW line successfully.

\section{Acknowledgements}

The authors wish to acknowledge David Wright, Paul Kimmins, Graeme Oliver and Tim Eggington, our industrial collaborators for their technical assistance on 
construction of WW simulation models and their co-operation in providing data, inhouse training and measurement on site in the factory.

The work has been carried out as part of the EPSRC E-IMRC at the University of Bath (GR/R67597/01).

\section{References}

Anon., 1985, Developing multi-function workers, Wire Journal International, 18 (1), $20-20$.

Bartholdi III, J.J., Leonid, D. and Eisenstein, D. D., 1999, Dynamics of two- and threeworker 'bucket brigade' production lines, Operations Research, 47 (3), 488-491.

Bartholdi III, J.J., Eisenstein, D. D. and Foley, R. D., 2001, Performance of bucket brigades when work is stochastic, Operations Research, 49 (5), 710-719.

Bischak, D. P., 1996, Performance of a manufacturing module with moving workers, IIE Transactions, 28, 723-733.

Black, J. T. and Schroer, B. J., 1997, Simulation of an apparel assembly cell with walking workers and decouplers, Journal of Manufacturing Systems, 12 (2), 170-180.

Bratcu, A. I. and Dolgui, A., 2005, A survey of the self-balancing production lines ('bucket brigades'), Journal of Intelligent Manufacturing, 16, 138-158. 
Celano, G., Costa, A., Fichera, S. and Perrone, G., 2003, Human factor policy testing in the sequencing of manual mixed model assembly lines, Computers \& Operations Research, 31 (1), 39-59.

Ghinato, P., Fujii, S. and Morita, H., 1998, Two effective methods for workforce reduction under lean production principles, $8^{\text {th }}$ International Flexible Automation and Intelligent Manufacturing Conference, pp. 703-714.

Groover, M. P., 2002, Fundamentals of modern manufacturing: materials, processes, and systems, pp. 860-911, (John Wiley \& Sons, Inc.,).

Hopp, J.W., Tekin, E. and Oyen, M. P. V., 2004, Benefits of skill chaining in serial production lines with cross-trained workers, Management Science, 50 (1), 83-98.

Mileham, A. R., Owen, G. W. and Pellegrin, W., F., 2000, The design and performance of walking-fitter assembly lines, Proceedings of $16^{\text {th }}$ International Conference on CAD/CAM, Robotics and Factories of the future, pp.325-332.

Nakade. K. and Ohno, K., 1997, Stochastic analysis of a U-shaped production line with multiple workers, Computers in Industrial Engineering, 33 (3-4), 809-812.

Nakada, K. and Ohno, K., 1999, An optimal worker allocation problem for a U-shaped production line, International Journal of Production Economics, 60 (1), 353-358. 
Nakade, K. and Ohno, K., 2003, Separate and carousel type allocations of workers in a U-shaped production line, European Journal of Operational Research, 145 (2), 403424.

Wang, Q. and Chatwin, C. R., 2003, Comparative dynamic performance of token passing and CSMA/CA LANs for a flexible manufacturing system, International Journal of Computer Integrated Manufacturing, 16 (6), 439-454.

Wang, Q., Owen, G. W. and Mileham, A. R., 2005, Assessing the effect of work time variations for a linear walking-fitter assembly line, Proceedings of the $3^{\text {rd }}$ International Conference on Manufacturing Research, Cranfield, UK.

Zavadlav, E., McClain, J. O. and Thomas, L. J., 1996, Self-buffering, self-balancing, self-flushing production lines, Management Science, 42 (8), 1151-1164. 


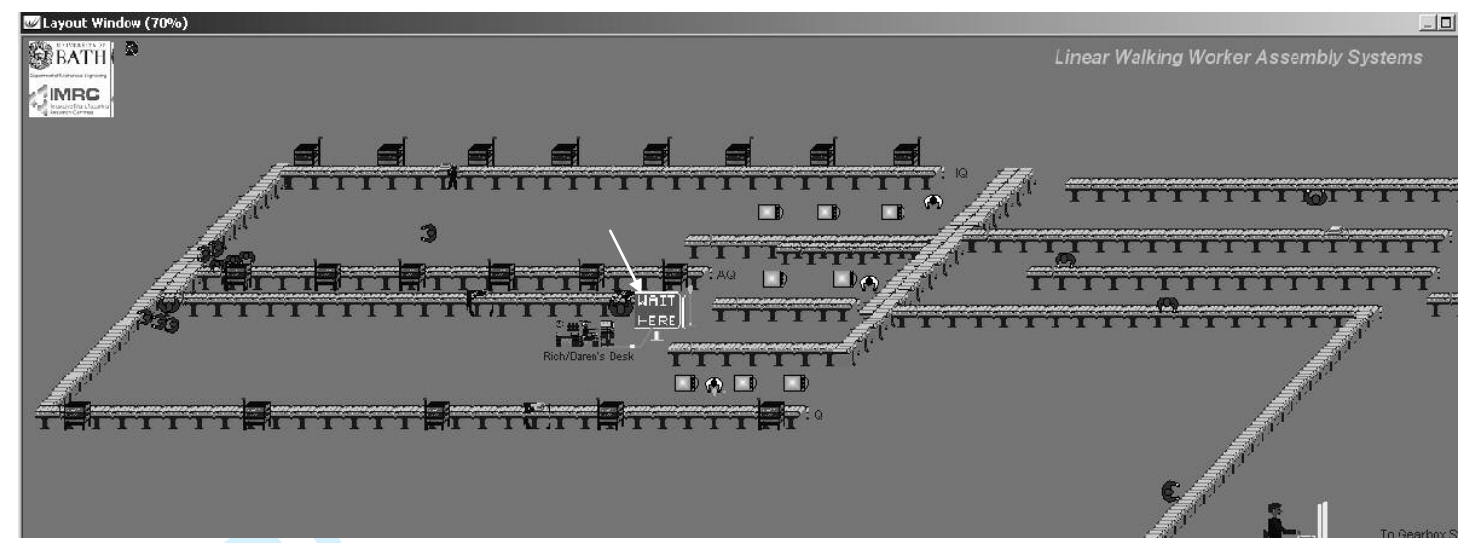

Figure 1. Layout of the linear WW assembly system (built by Witness ${ }^{\circledR}$ )

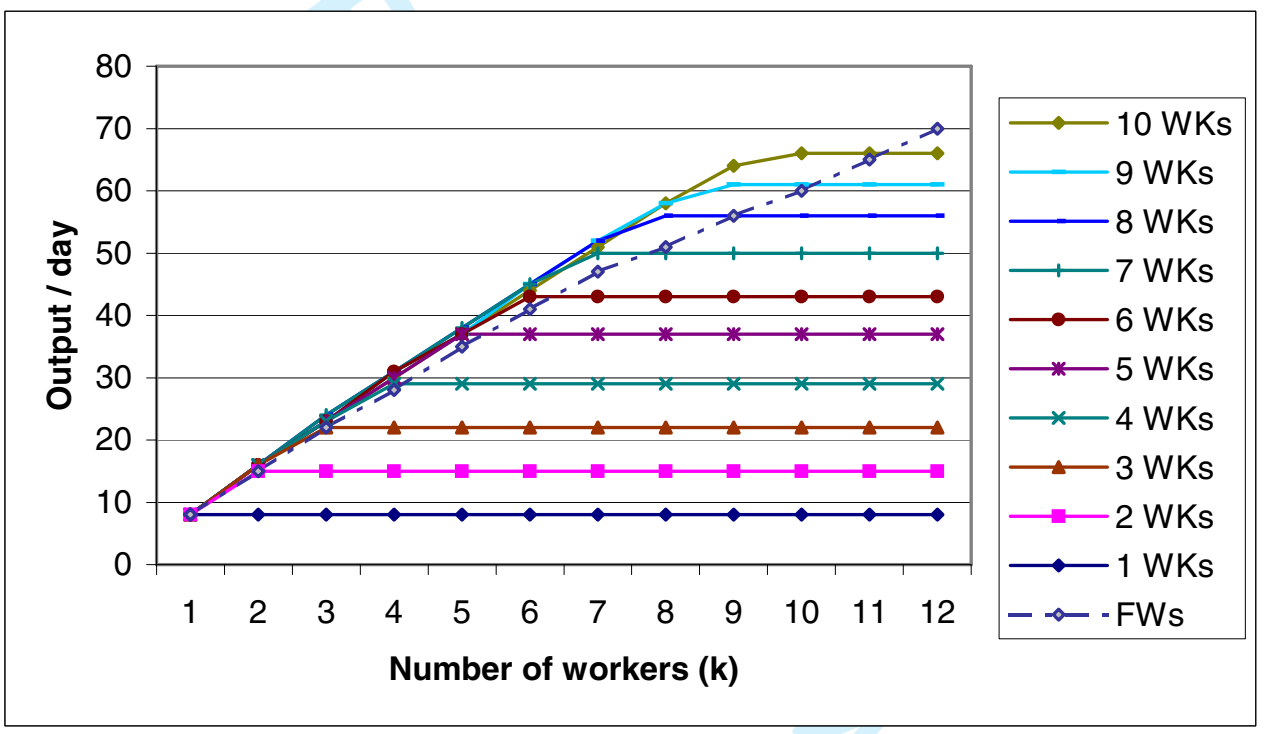

Figure 2. The overall output as a function of the number of walking or fixed workers (FWs) on an n-workstation (n WKs) line 


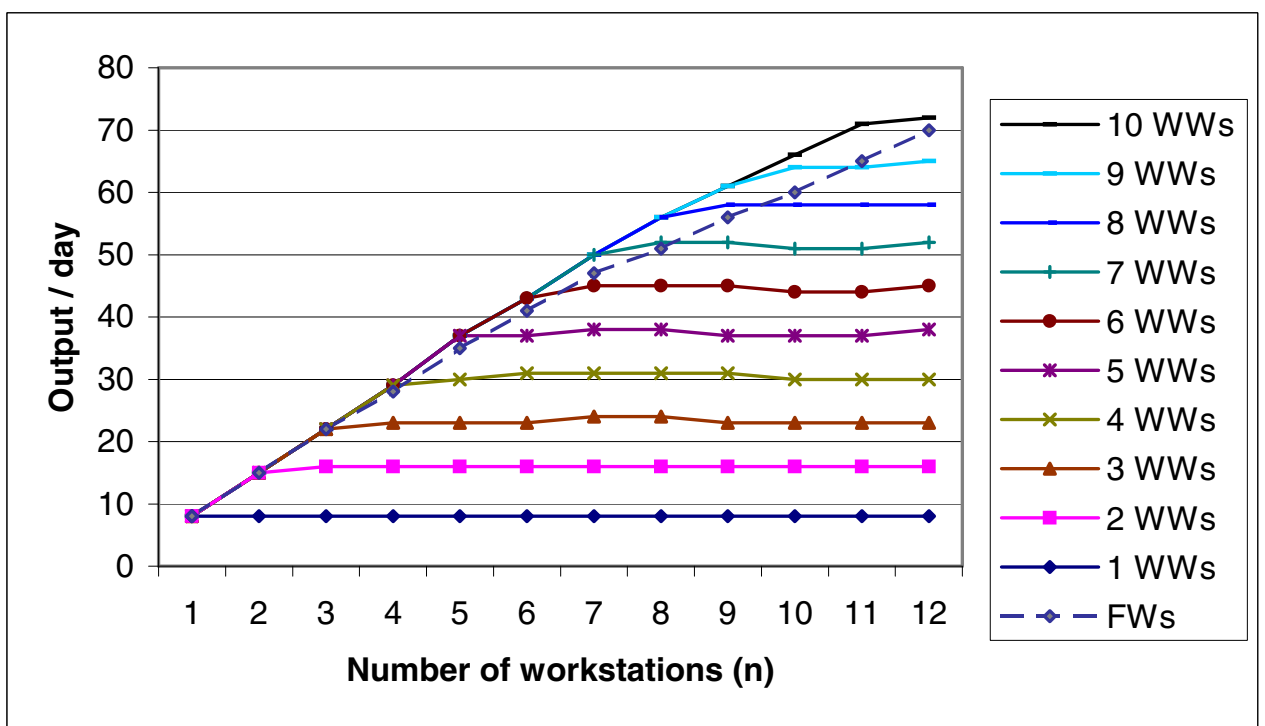

Figure 3. The overall output as a function of the number of workstations on a line with different numbers of walking workers (k WWs) or fixed workers (FWs)

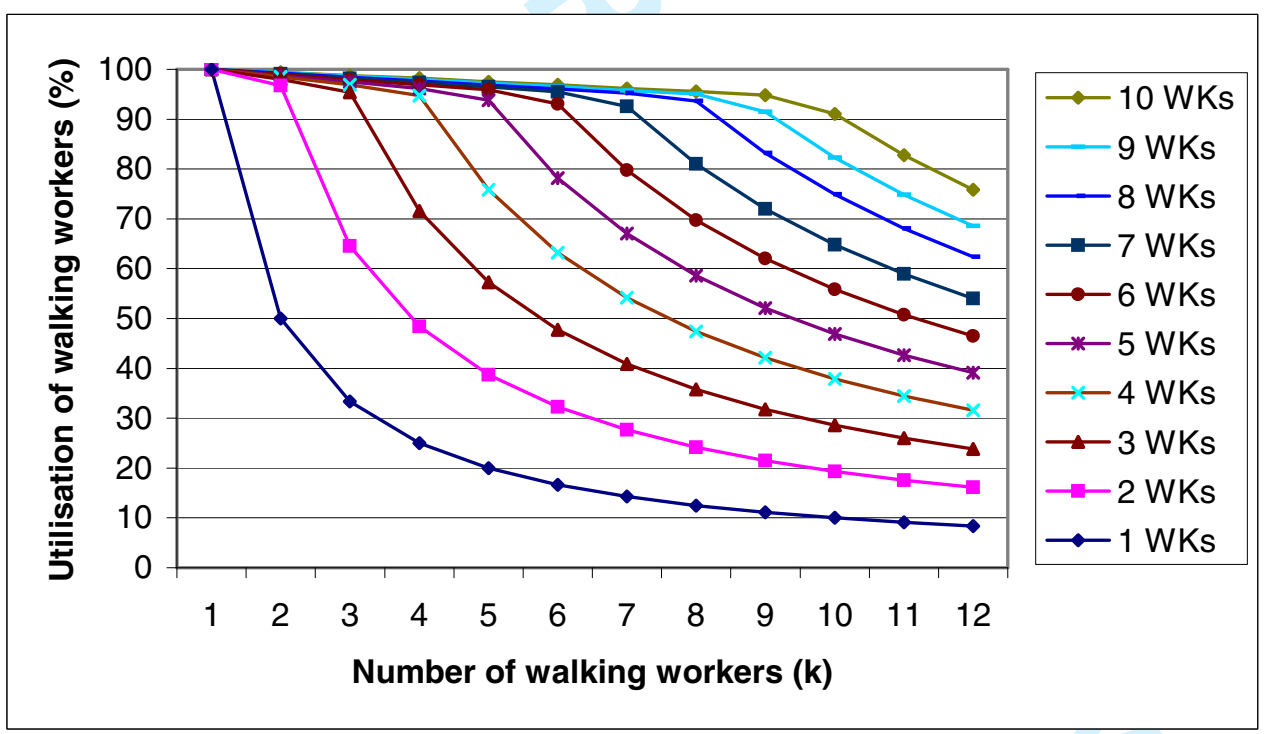

Figure 4. Labour utilisations versus numbers of walking workers on an n-workstation line (n WKs) 


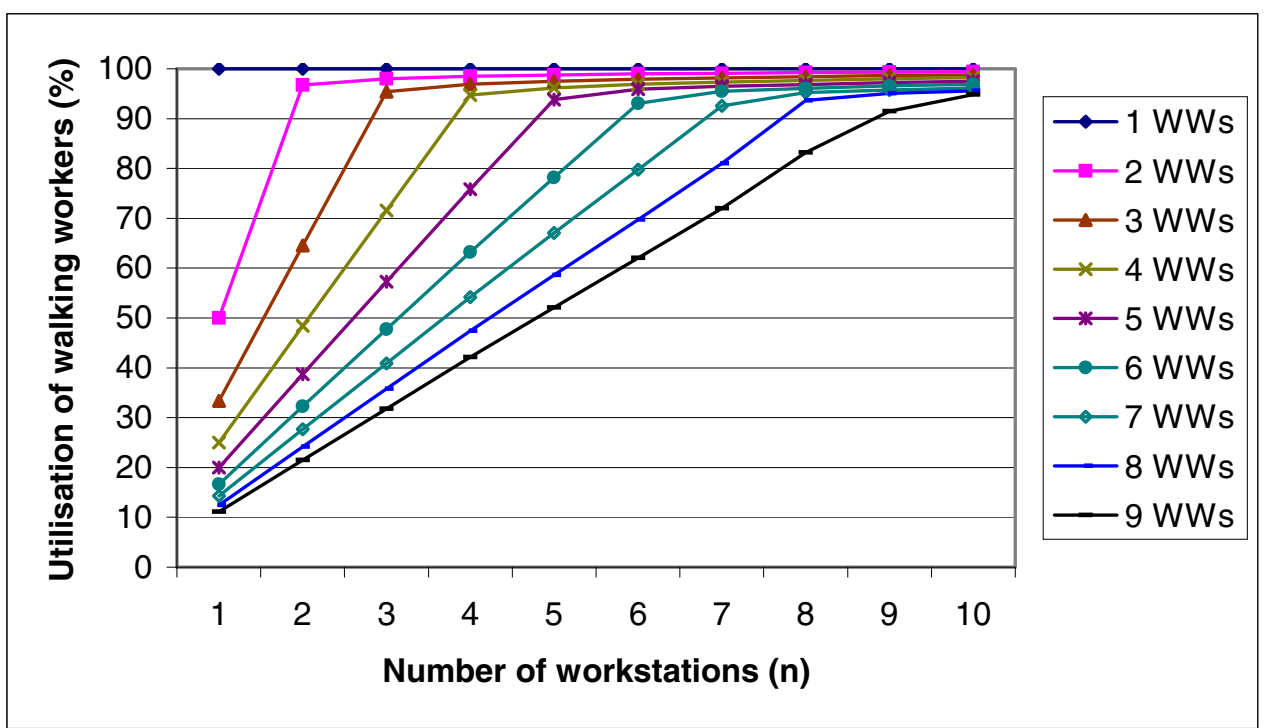

Figure 5. Labour utilisations versus numbers of workstations on a line with $\mathrm{k}$ walking workers (k WWs) 
Table 1. System parameters

\begin{tabular}{|c|c|c|c|c|}
\hline Line & $\begin{array}{c}\text { Product } \\
\text { range }\end{array}$ & $\begin{array}{c}\text { Number of } \\
\text { walking workers }\end{array}$ & $\begin{array}{c}\text { Number of } \\
\text { workstations }\end{array}$ & $\begin{array}{c}\text { Processing times (mean) } \\
\text { (minutes) }\end{array}$ \\
\hline A & A 1, 2 \& 3 & $4+$ & 8 & 5 \\
\hline B & B1, A 4 \& 5 & $4+$ & 6 & 8 for B1, 12 for A 4 \& 5 \\
\hline C & C1 & $4+$ & 5 & 10 \\
\hline
\end{tabular}

Table 2. The overall output as a function of the number of workstations for various numbers of walking workers

\begin{tabular}{|c|c|c|c|c|c|c|}
\hline \multirow{2}{*}{$\begin{array}{c}\text { Number of workstations } \\
(\mathrm{n})\end{array}$} & \multicolumn{6}{|c|}{ Outputs } \\
\cline { 2 - 7 } & 5 & 6 & 7 & 8 & 9 & 10 \\
\hline 5 & 37 & 37 & 37 & 37 & 37 & 37 \\
\hline 6 & 37 & 43 & 43 & 43 & 43 & 43 \\
\hline 7 & 38 & 45 & 50 & 50 & 50 & 50 \\
\hline 8 & 38 & 45 & 52 & 56 & 56 & 56 \\
\hline 9 & 37 & 45 & 52 & 58 & 61 & 61 \\
\hline 10 & 37 & 44 & 51 & 58 & 64 & 66 \\
\hline 11 & 37 & 44 & 51 & 58 & 64 & 71 \\
\hline 12 & 38 & 45 & 52 & 58 & 65 & 72 \\
\hline
\end{tabular}

Table 3. Comparison of line outputs between linear FW and WW lines

\begin{tabular}{|l|c|c|c|c|c|c|c|c|c|}
\hline No. of workers / workstations & 4 & 5 & 6 & 7 & 8 & 9 & 10 & 11 & 12 \\
\hline Outputs of FW lines & 28 & 35 & 41 & 47 & 51 & 56 & 60 & 65 & 70 \\
\hline Outputs of WW lines & 29 & 37 & 43 & 50 & 56 & 61 & 66 & 72 & 78 \\
\hline Surplus & 1 & 2 & 3 & 3 & 5 & 5 & 6 & 7 & 8 \\
\hline Increase & $3.6 \%$ & $5.7 \%$ & $7.3 \%$ & $6.4 \%$ & $9.8 \%$ & $8.9 \%$ & $10 \%$ & $11 \%$ & $11.4 \%$ \\
\hline Note: The results for k workers in an & norkstation line, where, k= n \\
\hline
\end{tabular}


Table 4. Comparison of highest blocking rates (\%) between linear FW and WW lines with varying levels in work time variations

\begin{tabular}{|c|c|c|c|c|c|c|}
\hline Number of & \multicolumn{7}{|c|}{$\begin{array}{c}\text { Work time variations } \\
\text { (Low }\end{array}$} \\
$\begin{array}{c}\text { workers or } \\
\text { workstations }\end{array}$ & \multicolumn{7}{|c|}{ High) } \\
\cline { 2 - 7 }$(\mathrm{n}=\mathrm{k})$ & \multicolumn{7}{|c|}{} & $\mathrm{FW}$ & WW & FW & WW & FW & WW \\
\hline & 1.86 & 0.80 & 2.87 & 2.16 & 4.16 & 3.52 \\
\hline 2 & 2.83 & 0.82 & 3.18 & 2.14 & 4.11 & 3.30 \\
\hline 3 & 4.88 & 1.52 & 5.55 & 4.02 & 6.92 & 5.75 \\
\hline 4 & 6.46 & 1.57 & 7.60 & 5.24 & 11.73 & 9.83 \\
\hline 5 & 7.99 & 2.19 & 9.56 & 7.16 & 15.19 & 13.00 \\
\hline 6 & 11.37 & 3.41 & 10.99 & 8.58 & 16.21 & 15.42 \\
\hline 7 & 15.80 & 9.50 & 15.71 & 13.05 & 23.17 & 21.31 \\
\hline 8 & 19.57 & 10.67 & 18.05 & 14.93 & 23.14 & 21.69 \\
\hline 9 & 21.77 & 14.17 & 22.57 & 18.47 & 26.83 & 22.36 \\
\hline 10 & & & & & & \\
\hline
\end{tabular}

